\title{
Does Extracorporeal Shock Wave Lithotripsy Before Retrograde Intrarenal Surgery Complicate the Surgery for Upper Ureter Stone? The Results of RIRSearch Group.
}

\author{
Cenk Murat Yazıcı ${ }^{1}$, Duygu Siddikoglu², Onder Cinar ${ }^{3}$, Oktay Ozman $^{2}$, Cem Basatac $^{2}$, \\ Murat Akgul ${ }^{1}$, Eyup Burak Sancak ${ }^{2}$, Bulent Onal ${ }^{4}$, and Haluk Akpinar ${ }^{2}$ \\ ${ }^{1}$ Namik Kemal University Faculty of Medicine \\ ${ }^{2}$ Affiliation not available \\ ${ }^{3}$ Zonguldak Bulent Ecevit University \\ ${ }^{4}$ Istanbul Universitesi-Cerrahpasa
}

November 12, 2020

\begin{abstract}
Aims To evaluate the effect of pre-RIRS ESWL on the efficiency and safety of RIRS in the treatment of proximal ureter stones. Methods The patients in the study population were divided into 2 groups. Group-1 was composed of patients who had undergone ESWL for proximal ureter stones before RIRS, and Group-2 was composed of patients who directly underwent RIRS without any prior ESWL. The clinical and demographic properties of the patients were analysed in the RIRSearch database. The operative outcomes, peroperative complications, postoperative complications, hospitalization time and the stone-free rates were compared between the groups. Results There were 56 patients in Group-1 and 95 patients in Group-2. The demographic and clinical properties were similar between the groups. The stone-free rates, peroperative complications and postoperative complications were also similar between the groups; however, the fluoroscopy time was significantly higher in Group-1 ( $\mathrm{p}=.043$ ). The cut-off duration of 10 weeks between ESWL and RIRS had reasonable/favourable discriminating ability, with a $51 \%$ sensitivity and $88 \%$ specificity rate for stone-free status. Conclusion Performing ESWL on the proximal ureter stones before RIRS did not change the efficacy and safety of RIRS. The time between the patient's last ESWL session and RIRS had a predictive value for stone-free status, but did not have any effect on complications.
\end{abstract}

Does Extracorporeal Shock Wave Lithotripsy Before Retrograde Intrarenal Surgery Complicate the Surgery for Upper Ureter Stone? The Results of RIRSearch Group.

Running Title: Retrograde Intrarenal Surgery After Extracorporeal Shock Wave Lithotripsy for Upper Ureteric Stones.

Cenk Murat Yazici ${ }^{1}$, Duygu Siddikoglu ${ }^{2}$, Onder Cinar ${ }^{3}$, Oktay Ozman $^{4}$, Cem Basatac ${ }^{5}$, Murat Akgul $^{1}$, Eyup Burak Sancak ${ }^{6}$, Bulent Onal ${ }^{7}$, Haluk Akpinar ${ }^{5}$

(1) Namık Kemal University, School of Medicine, Department of Urology, Tekirdag, Turkey.

(2) Canakkale Onsekiz Mart University, School of Medicine, Department of Statistics, Canakkale, Turkey.

(3) Zonguldak Bulent Ecevit University, School of Medicine, Department of Urology, Zonguldak, Turkey.

(4) Gaziosmanpasa Training Hospital, Clinic of Urology, Istanbul, Turkey. 
(5) Gayrettepe Florence Nightingale Hospital, Department of Urology, Istanbul, Turkey.

(6) Canakkale Onsekiz Mart University, School of Medicine, Department of Urology, Canakkale, Turkey.

(7) Istanbul University Cerrahpasa School of Medicine, Department of Urology, Istanbul, Turkey.

Acknowledgement: None.

Declerations: The authors have no relevant financial or non-financial interests to disclosure.

Funding: The authors did not recieve support from any organization fort he submitted work.

Financial interests: The authors declare they hane no financial interests.

Conflict of interest: All the authors declare no conflict of interest

Ethics approval: Approved by Namık Kemal University Ethical Committee, Tekirdag, Turkey. The procedures used in this study adhere to the tenets of the Declaration of Helsinki.

Author contributions: $\mathrm{CMY}, \mathrm{HMA}, \mathrm{EBC}, \mathrm{CB}, \mathrm{OC}, \mathrm{BO}, \mathrm{HA}$ and $\mathrm{OO}$ collected the original data and contributed to design of the study. DS performed the statistical analysis of the data. CMY, BO and HA performed the writing of the article.

Avaibility of data and material: All the data related to study are available in RIRsearch data base.

Abstract

Aims

To evaluate the effect of pre-RIRS ESWL on the efficiency and safety of RIRS in the treatment of proximal ureter stones.

Methods

The patients in the study population were divided into 2 groups. Group-1 was composed of patients who had undergone ESWL for proximal ureter stones before RIRS, and Group-2 was composed of patients who directly underwent RIRS without any prior ESWL. The clinical and demographic properties of the patients were analysed in the RIRSearch database. The operative outcomes, peroperative complications, postoperative complications, hospitalization time and the stone-free rates were compared between the groups.

\section{Results}

There were 56 patients in Group-1 and 95 patients in Group-2. The demographic and clinical properties were similar between the groups. The stone-free rates, peroperative complications and postoperative complications were also similar between the groups; however, the fluoroscopy time was significantly higher in Group-1 ( $\mathrm{p}=.043)$. The cut-off duration of 10 weeks between ESWL and RIRS had reasonable/favourable discriminating ability, with a $51 \%$ sensitivity and $88 \%$ specificity rate for stone-free status.

\section{Conclusion}

Performing ESWL on the proximal ureter stones before RIRS did not change the efficacy and safety of RIRS. The time between the patient's last ESWL session and RIRS had a predictive value for stone-free status, but did not have any effect on complications.

Keywords: retrograde intrarenal surgery, extracorporeal shock wave lithotripsy, efficacy, safety, complications, stone-free.

What is already known about this topic? 
The effect of presurgical ESWL on the efficacy and safety of semirigid ureteroscopy was shown in some studies. These studies reported that the presurgical ESWL did not complicate the semirigid ureteroscopy and did not increase the complication rate. On the other hand, with the evolution of technology retrograde intrarenal surgery became a standard procedure for patients with upper ureter stones. There is a significant gap in the literature defining the effect of pre-RIRS ESWL on the efficacy and safety of at RIRS for upper ureter stone treatment.

\section{What does this article add?}

The results showed that ESWL before the RIRS did not complicate the surgery but significantly increased the peroperative fluoroscopy time. The duration between the last ESWL session to the RIRS was a predictor for stone free-rates. The number of ESWL sessions before the RIRS was a predictor of operation time. According to our knowledge this article is the first article documenting the effect of pre-RIRS ESWL on the efficacy and safety of RIRS.

\section{Introduction}

The evolutional history of retrograde intrarenal surgery (RIRS) dates back to the 1970s, but the clinical usage of this technique has gained increasing popularity in the last 2 decades [1]. The functional developments of flexible ureteroscopes, lasers and ancillary equipment have led RIRS to become a significant surgical technique for the treatment of upper urinary system stones [2]. Due to these technological advancements, the guidelines for the treatment of urinary system stone disease have been frequently revised.

Despite these technological advancements, extracorporeal shock wave lithotripsy (ESWL) still maintains its importance for the treatment of upper urinary system stones less than $2 \mathrm{~cm}$. in diameter. On the other hand, recent studies reporting the trends of urolithiasis treatment have shown a significant increase in the use of ureteroscopy compared to ESWL [3-5]. RIRS and ESWL are the 2 main treatments for proximal ureter stones. The European Association of Urology (EAU) guidelines recommend ureteroscopy as the first-line treatment for proximal ureter stones $>10 \mathrm{~mm}$, and ureteroscopy or ESWL for proximal ureter stones $<10$ mm [6]. According to the American Urology Association and Endourology Society guidelines, ureteroscopy is recommended as the treatment modality for proximal ureter stones of any size $[7,8]$. The efficacy and the safety of RIRS has led this procedure to replace the role of ESWL in the treatment of proximal ureter stones. On the other hand, expensive and fragile surgical equipment make this surgery a costly procedure. For this reason, there are several clinics that perform ESWL as the first-line treatment of proximal ureter stones and prefer RIRS as the second-line treatment for patients in which ESWL is not successful.

ESWL has proven to be a safe and effective method for the treatment of urinary system stones; however, it may lead to tissue damage, that is localized around the target zone. Animal studies have documented that ESWL causes torn vessels with platelet aggregation; vacuolization to complete necrosis of the epithelium and vascular smooth tissue; and aggregation of red blood cells and leukocytes in the interstitial space. The micro-environmental changes lead to tissue oedema and an inflammatory reaction around the target stone [9]. This reaction may also occur at the ureter wall around the stone, and may cause the stone to impact at the ureteric mucosa. The traumatic effect of ESWL on the ureteral wall and mucosal oedema may be considered a complication of RIRS that affects the efficiency and safety of this procedure.

We hypothesized that performing ESWL on proximal ureter stones before RIRS may be a disadvantage for the efficiency and safety of the surgery. In order to determine the validity of our hypothesis, we evaluated the effect of pre-RIRS ESWL on the efficiency and safety of RIRS in the treatment of proximal ureter stones.

\section{Materials and Methods}

With the approval of the local ethics committee (approval number 2020.193.08.02), patients who underwent RIRS for the treatment of proximal ureter stones between 2014 and 2020 at 4 referral centres were retrospectively included in the study. The proximal ureter was defined as the ureteral portion between the ureteropelvic junction and the upper border of the iliac crest. Patients younger than 18 years of age; patients with a renal anatomical anomaly, bleeding tendency, solitary kidney, multiple stones (even at the proximal 
ureter); and patients who had undergone previous ipsilateral ureteric or renal surgery were excluded from the study.

The patients in the study population were divided into 2 groups. Group 1 was composed of patients who had undergone ESWL for proximal ureter stone before RIRS, and Group 2 was composed of patients who directly underwent RIRS without any prior ESWL. RIRS was performed by urologists from the 4 centres who had a minimum of 5 years' experience. The clinical and demographic properties of the patients, including age, gender, body mass index, preoperative serum creatinine level, preoperative renal hydronephrosis, number of ESWL sessions, duration between the last ESWL session and RIRS and stone-related parameters, such as stone volume, stone density and location, were analysed at our database. All patients underwent preoperative evaluation, which included a detailed anamnesis, physical examination and routine laboratory studies, including serum creatinine, urinalysis and a urine culture, and a preoperative radiologic evaluation with non-contrast computed tomography (NCCT). The stone volume was calculated based on the NCCT images using the formula as follows: length $\times$ width $\times$ height $\times 0.523\left(\mathrm{~mm}^{3}\right)$. Stone density was measured by the mean value of three Hounsfield unit (HU) measurements that were taken from the centre, sub-centre and peripheral regions of the maximum stone length in the axial NCCT image. The operative outcomes (operation time and fluoroscopy time), peroperative complications, postoperative complications, hospitalization time and the stone-free rates after RIRS were compared between the groups. The stone-free status was defined as: no evidence of residual stones or remaining residual fragments of $<3 \mathrm{~mm}$ on the first month postoperative NCCT images.

\section{Surgical technique}

All patients provided written informed consent before the surgery. As a standard procedure, pre-surgical urine cultures were obtained and positive cultures were treated according to antibiogram. Second-generation cephalosporins were utilized for preoperative antibiotic prophylaxis. All of the surgeries were performed under general anaesthesia. After the cystoscopic evaluation of the bladder, a retrograde pyelography was performed to evaluate the upper urinary system. A 0.035-inch safety guidewire (Sensor ${ }^{\circledR}$, Boston Scientific, Marlborough, MA, USA) was routinely placed. Then, the ureteral access sheath (UAS; 10-12 Fr. Bi-Flex, Rocamed, Monaco or 11/13 Fr. Boston Scientific, Marlborough, MA, USA) was inserted over the working guidewire and placed just below the proximal ureter stone under fluoroscopic guidance. The flexible ureteroscope (MARKA) was inserted through the UAS, and a holmium: YAG laser with a $272 \mu \mathrm{m}$ laser fibre was used to fragment the stones. The laser energy and pulse frequency were varied based on the stone burden, stone density and the surgeon's preference. Stone fragments $>2 \mathrm{~mm}$ were extracted using a nitinol basket catheter (Dakota ${ }^{\circledR}$, Boston Scientific, Marlborough, MA, USA). A 4.7 Fr. double J stent was inserted into the urinary system and left in place for 1 to 4 weeks according to the surgeon's preference. During followup, all patients were evaluated using kidney-ureter-bladder radiography at the postoperative first day and low-dose CT in the postoperative first month.

\section{Statistical analysis}

Categorical variables were expressed as frequencies and percentages (\%), and continuous variables were expressed as mean \pm standard deviation (SD). The Shapiro-Wilk test was used to assess the normality assumption for the continuous variables. The Student's t-test or Mann-Whitney U test was used between groups, whether the statistical hypotheses were fulfilled or not. The Chi-squared test was used to compare the distribution of categorical variables in groups. Simple logistic regression was used for bivariate analysis to identify individual associations with stone-free status (Yes/No), hospitalization time (1 day versus more than 1 day) and postoperative complications (Yes/No). A p value of $<.25$ was accepted as significant in the selection of the factors whose efficacy would be assessed with multiple logistic regression analysis, which was performed stepwise. Significant results are presented as odds ratios (ORs) and 95\% confidence interval (CI). Multiple regression was run to predict operation time. Non-significant covariates were removed from the model. There was linearity as assessed by a plot of studentized residuals against the predicted values. The area under the receiver operating curve (AUC) was calculated. All statistical analyses were performed using SPSS 19.0 software (IBM Corp., Armonk, NY, USA). All statistical tests had a statistical significance 
level of $\mathrm{p}$ value $<.05$ (two-tailed).

\section{Results}

There were 56 patients in Group 1 and 95 patients in Group 2. The demographic characteristics of the patients and the stone-related variables were similar between the groups. The preoperative serum creatinine level, the rate of preoperative double J stenting and the rate of hydronephrosis were also similar between the groups. The mean number of ESWL sessions and the mean duration between the last ESWL session and RIRS in Group 1 were $2.9 \pm 1.0$ times and $68.4 \pm 69.9$ days, respectively (Table 1 ).

The stone-free rates of Group 1 and Group 2 were $94.6 \%$ and $93.7 \%$, respectively $(\mathrm{p}=.893)$. The mean operation time was $63.3 \pm 24.5 \mathrm{~min}$ for Group 1 and $63.4 \pm 25.6 \mathrm{~min}$ for Group $2(\mathrm{p}=.997)$. The fluoroscopy screening time and hospitalization time were significantly higher in the Group 1 patients. Peroperative and postoperative complications were similar between the groups (Table 2). All of the peroperative complications in both groups were classified as Grade 1 on the Clavien-Dindo classification scale, which included mucosal erosion and mild bleeding. A total of $7(12.5 \%)$ patients in Group 1 had postoperative complications, including postoperative fever that required antibiotherapy. Among the patients in Group 2, 10 (10.5\%) had postoperative complications, including fever (8 patients) and urosepsis (2 patients).

In order to evaluate the possible predictive effect of ESWL on the efficacy and safety of RIRS for proximal ureter stone treatment, a model of variables composed of age, sex, number of ESWL sessions, the duration between ESWL and RIRS, stone density, presence of hydronephrosis and stone volume was formed. According to this group analysis, the duration between ESWL and RIRS (in weeks) was found to be a predictive factor for stone-free status. On the other hand, the AUC (0.662) was found to be statistically non-significant $(\mathrm{p}=$ $.126)$ and the Youden index was low (0.400); hence, it was suggested that the time from ESWL to RIRS had no discriminatory ability. Nonetheless, all patients who underwent RIRS after duration of 10 weeks postESWL were stone-free, and the cut-off duration of 10 weeks from ESWL to RIRS had reasonable/favourable discriminating ability with $51 \%$ sensitivity and $88 \%$ specificity. The OR for the duration between ESWL and RIRS was 1.27 (95\% CI: 1.11-1.44) for each week. The relation between the model variables and the operation time, hospitalization time and complications are given in Table 3.

\section{Discussion}

RIRS is an emerging surgical technique for the treatment of upper urinary system stone disease. Despite its invasive nature, the preference rate of RIRS has increased significantly [2]. On the other hand, many urologists prefer ESWL as the first-line treatment of proximal ureteral stone disease. It is scientifically reasonable that the EAU guidelines recommend ESWL and ureteroscopy as equally valid for the treatment of proximal ureter stones $<10 \mathrm{~mm}[6]$. For that reason, many patients undergo ESWL for proximal ureteral stone disease, and RIRS becomes the second-line treatment modality for patients in which ESWL is unsuccessful.

Although ESWL is defined as a non-invasive procedure, it has some destructive effects on tissues [10]. Studies have demonstrated that the shock waves might lead to microvascular damage and shear effect at the tissue around the stones $[11,12]$. The traumatic effect of ESWL has also been clinically observed. Nearly all ESWL sessions cause microscopic or macroscopic haematuria. Experimental studies have demonstrated that uroepithelial irritation was not the only reason for hematuria, the main reason was microvascular damage and local trauma [11]. Histomorphologic studies showed that shock waves induce torn vessels with platelet aggregation; vacuolization to complete necrosis of the epithelium and vascular smooth muscle; and aggregation of red blood cells and leucocytes in the interstitial space [13]. Although these findings have been shown in the renal parenchyma and papilla, the ureteral wall might also be exposed to similar reactions after ESWL treatment of ureteric stones. Ureteral stricture is a devastating complication of ESWL, which might be the result of an extreme reaction that occurs due to tissue damage from the shock waves [14].

One of the main questions of our study was: "Does the presence of pre-RIRS ESWL complicate the RIRS procedure?" The peroperative variables, such as operation time and peroperative complication rates, were similar between the groups. The postoperative variables, such as postoperative complication rates and hos- 
pitalization time, were also similar between the groups. These data revealed that performing ESWL before RIRS did not complicate the surgical procedure for proximal ureteral stone disease. On the other hand, the fluoroscopy screening time was nearly twice as long, with a significant difference in patients who had undergone ESWL before RIRS. This data showed that surgeons had to use more fluoroscopy during RIRS for patients who have undergone ESWL before the surgery. The importance of radiation exposure during the surgery is a well-known phenomenon by surgeons, and all surgeons try to obey the ALARA (As Low As Reasonably Achievable) principles. For that reason, the significant difference in radiation time between the groups might demonstrate that the surgeons of our cohort had to be more cautious during RIRS performed after ESWL. This finding was an important output of our study. Although the operation time and peroperative complication rates showed that pre-RIRS ESWL did not complicate the surgical procedure, surgeons had to use more radiation during the procedure for patients who had previously undergone ESWL.

Another question that we tried to answer in this study was: "Does the presence of pre-RIRS ESWL affect the stone-free rates of RIRS?" The stone-free rates were similar between the groups, which showed that the performance of ESWL before the surgery did not affect the surgical success. On the other hand, the time from the ESWL and RIRS had a predictive effect on the success rate of RIRS. The stone-free rates of RIRS for proximal ureter stones increased as the duration between ESWL and RIRS increased. The authors found that the OR for stone-free status was 1.27 for each preceding week, and the threshold time was 10 weeks with $51 \%$ sensitivity and $88 \%$ specificity. To our knowledge, this study is the first in the literature documenting the relation between stone-free status and the duration between ESWL and RIRS. As the proximal ureter stones may lead to hydronephrosis and a heightened risk for renal dysfunction, it may not be logical to wait for 2 months after the ESWL to perform RIRS. On the other hand, this data may be important to predict the success of RIRS. For the patients who have very recently undergone ESWL, inserting a double J stent and postponing the surgery for a while may be a solution to increase the stone-free rates of patients.

There have been some studies evaluating the predictive factors of the efficacy and safety of semirigid ureteroscopy for the treatment of upper ureteral stones. The variables related to stone migration, such as the length and diameter of the proximal ureter portion, stone size and surgeon's experience, were the main predictors for the success rate of semirigid ureteroscopy [15]. Stone migration has limited importance for RIRS because the flexible nature of the ureteroscopes let the surgeons follow the migrated stone and complete the surgery. Although semirigid ureteroscopy and RIRS have similarities in terms of technical principles, they are totally different in terms of surgical procedure. For this reason, it is hard to compare the predictive factors for the success of semirigid ureteroscopy with RIRS.

The retrospective design and relatively limited study population were the main limitations of our study. However, the study data were obtained from the RIRSearch database, which was prospectively created with detailed evaluation. Another limitation was the lack of ESWL standardization in our study group. The patients had undergone ESWL sessions in different institutions, which impacted the standardization of the procedure. However, it is a reality that surgeons have to perform RIRS on patients who have undergone ESWL in different clinics without standardization. We believe that this study is valuable because it represents the first investigation evaluating the effect of pre-RIRS ESWL on the results of RIRS in the treatment of proximal ureter stones.

\section{Conclusion}

In conclusion, performing ESWL on proximal ureter stones before RIRS did not change the efficacy and safety of RIRS. The presence of ESWL before RIRS significantly increased the peroperative fluoroscopy time. Although the time between the last ESWL session and RIRS had a predictive value for stone-free status, it did not have any effect on complications. As the duration between the ESWL session and RIRS increased, the stone-free rates also increased. The number of ESWL sessions was related with operation time with a positive relationship.

\section{Acknowledegements: None}

Conflicts of interest: The authors have declared that they have no conflicts of interest. 


\section{Funding: None}

\section{References}

1.Takayasu H, Aso Y, Takagi T, Go T; 1971; Clinical application of fiber-optic pyeloureteroscope. Urol Int 26:97-104.https://doi.org/10.1159/000279719

2- Rodríguez-Monsalve Herrero M, Doizi S, Keller EX, De Coninck V, Traxer O; 2018; Retrograde intrarenal surgery: An expanding role in treatment of urolithiasis. Asian J Urol 5(4):264273.https://doi.org/10.1016/j.ajur.2018.06.005

3-Oberlin DT, Flum AS, Bachrach L, Matulewicz RS, Flury SC; 2015; Contemporary surgical trends in the management of upper tract calculi. J Urol 193:880.https://doi.org/10.1016/j.juro.2014.09.006

4-Lee MC, Bariol SV; 2011; Evolution of stone management in Australia. BJU Int 108(2):2933.https://doi.org/10.1111/j.1464-410X.2011.10695.x

5- Doizi S, Raynal G, Traxer O; 2015; Evolution of urolithiasis treatment over 30 years in a French academic institution. Prog Urol 25:543-8.https://doi.org/10.1016/j.purol.2015.05.002

6-EAU Guidelines on Urolithiasis. Professionals S-O. Urolithiasis [Internet]. Uroweb. [cited 2018 Apr 4]. Available from:http://uroweb.org/guideline/urolithiasis/.

7- Assimos D, Krambeck A, Miller NL, Monga M, Murad MH, Nelson CP et al; 2016; Surgical management of stones: american urological association / endourological society guideline, PART I. J Urol 196:115360.https://doi.org/10.1016/j.juro.2016.05.090

8- Assimos D, Krambeck A, Miller NL, Monga M, Murad MH, Nelson CP et al; 2016; Surgical management of stones: american urological association/endourological society guideline, PART II. J Urol 196:11619.https://doi.org/10.1016/j.juro.2016.05.091

9- Evan AP, Willis LR, Connors B, Reed G, McAteer JA, Lingeman JE; 1991; Shock wave lithotripsy-induced renal injury. Am J Kid Dis 17:445-450.https://doi.org/10.1016/S0272-6386(12)80639-1

10- Cui X, Ji F, Yan H, Ou TW, Jia CS, He XZ et al; 2015; Comparison between extracorporeal shock wave lithotripsy and ureteroscopic lithotripsy for treating large proximal ureteral stones: a meta-analysis. Urology 85(4):748-56.https://doi.org/10.1016/j.urology.2014.11.041

11- McAteer JA, Evan AP; 2008; The Acute and Long-Term Adverse Effects of Shock Wave Lithotripsy. Semin Nephrol 28(2): 200-213.https://doi.org/10.1016/j.semnephrol.2008.01.003

12- Evan AP et al; 2002; Kidney damage and renal functional changes are minimized by waveform control that suppresses cavitation in SWL. J Urol 168:1556-1562.https://doi.org/10.1097/01.ju.0000024272.27628.1a

13- Yamauchi T, Tsukamoto T, Mori Y, Sugiyama K, Fujioka T; 2003; Ureteral Stricture After ESWL for Ureteral Calculi. Nihon Hinyokika Gakkai Zasshi 94(1):8-14.https://doi.org/10.5980/jpnjurol1989.94.8

14- Miroglu C, Horasanli K, Tanriverdi O, Altay B, Gumus E; 2006; Operative failure during ureteroscopic pneumatic lithotripsy: factors affecting successful outcome. Urol Int. 77(2):14851.https://doi.org/10.1159/000093910

15- Schuster TG, Hollenbeck BK, Faerber GJ, Wolf JS Jr; 2001; Complications of ureteroscopy: analysis of predictive factors. J Urol 166: 538-540.https://doi.org/10.1016/S0022-5347(05)65978-2

\section{Hosted file}

Table1IJCPESL.pdf available at https://authorea.com/users/375406/articles/492651-doesextracorporeal-shock-wave-lithotripsy-before-retrograde-intrarenal-surgery-complicatethe-surgery-for-upper-ureter-stone-the-results-of-rirsearch-group

\section{Hosted file}


table2IJCPESL.pdf available at https://authorea.com/users/375406/articles/492651-doesextracorporeal-shock-wave-lithotripsy-before-retrograde-intrarenal-surgery-complicatethe-surgery-for-upper-ureter-stone-the-results-of-rirsearch-group

\section{Hosted file}

table3IJCP-ESL.pdf available at https://authorea.com/users/375406/articles/492651-doesextracorporeal-shock-wave-lithotripsy-before-retrograde-intrarenal-surgery-complicatethe-surgery-for-upper-ureter-stone-the-results-of-rirsearch-group 